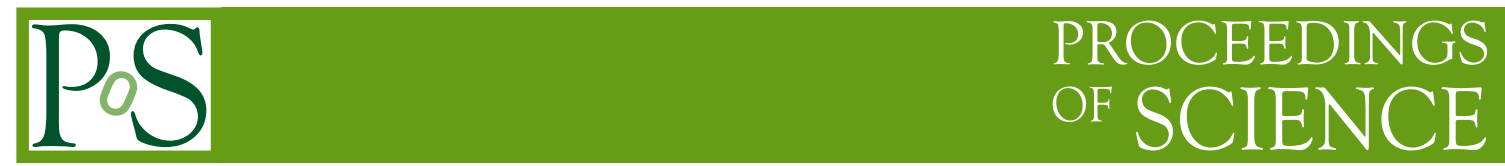

\title{
Micropattern Gas Detectors
}

\section{Jochen Kaminski*}

University of Bonn, Germany

E-mail: kaminski@physk.uni-bonn.de

An overview of Micropattern Gas Detectors is given. Recent progress of detector research, especially in the context of Micromegas and Gas Electron Multipliers, is discussed. A particular emphasis is put on the pixelized readout with a dedicated ASIC nameed Timepix, where new results with both gas amplification stages atop this chip are presented.

17th International Workshop on Vertex Detectors

July 28 - August 1, 2008

Utö Island, Sweden

\footnotetext{
* Speaker.
} 
a)

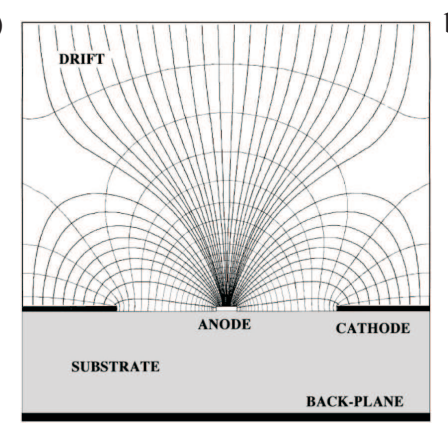

b)

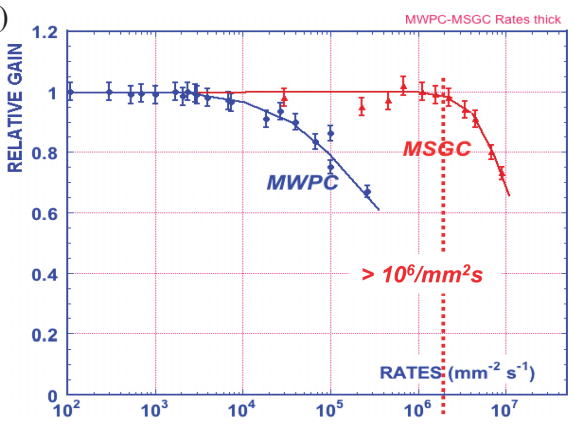

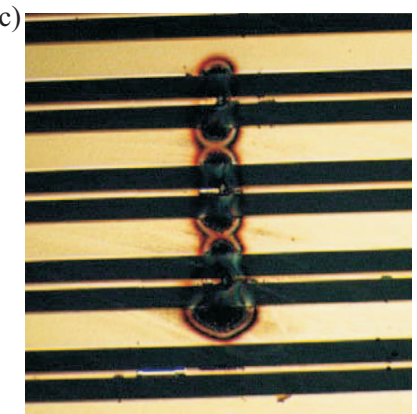

Figure 1: Microstrip Gas Chambers: a) schematic drawing and electric field configuration of a MSGC, b) comparison of MWPC and MSGC with respect to their rate capability, c) photograph of damaged MSGC.

\section{Overview over Historic Progress in Gaseous Detector Development}

In 1908 E. Rutherford and H. Geiger published the first paper on wire counters describing the use of gas amplification in the vicinity of a wire for their study of natural radioactivity.[1] During the following century a vast number of papers were published testifying the rapid progress in turning gaseous detectors in a widely used tool. Interestingly enough major innovations were published in intervals of roughly 20 years. In $1928 \mathrm{H}$. Geiger and W. Müller could show, that their improved wire counter was sensitive to single electrons.[2] Then an exact charge measurement was made possible, when proportional tubes were made to work around 1945. G. Charpak and his collaborators made wire chambers attractive for large area applications by introducing multiwire proportional chambers for which he was honored with the 1992 Nobel Prize for Physics.[3] The age of micropattern gas detectors (MPGDs) was introduced by A. Oed, when he demonstrated the first functional Microstrip Gas Chambers (MSGCs).[4] Finally, in 2004, a few years early, it was demonstrated that micro pattern gas detectors could be readout with a highly pixelized ASIC,[5][6] resolving single electrons drifting in the gas.

\section{Micropattern Gas Detectors}

Since the first publication on Micropattern Gas Detectors 20 years ago, an extensive research program was started to understand the new detector generation and to optimize it for the needs of high energy physics or other applications. A more detailed overview can be found in reference [7]. In the course of these studies many desired features could be demonstrated, and many new designs were suggested to overcome shortcomings.

In the 1990s the main focus of development studies was placed on the aforementioned MSGCs, which shall be explained as an example in more detail. They consist of a pattern of alternating thin anode strips $(\mathscr{O}(10 \mu \mathrm{m}))$ and wider cathode strips with a pitch in the order of $100 \mu \mathrm{m}$ (see figure 1a). With the help of photolithographic processes the fine metal structures were applied on a glass substrate. If a sufficiently large voltage difference is applied between the anode and cathode strips, strong local dipole fields are created within the vicinity of the anode strip. In these fields gas amplification due to avalanche processes take place and the electron signal can be decoupled and read out from the anode strip. Thanks to the small separation of the cathode and the anode the ions 
a)

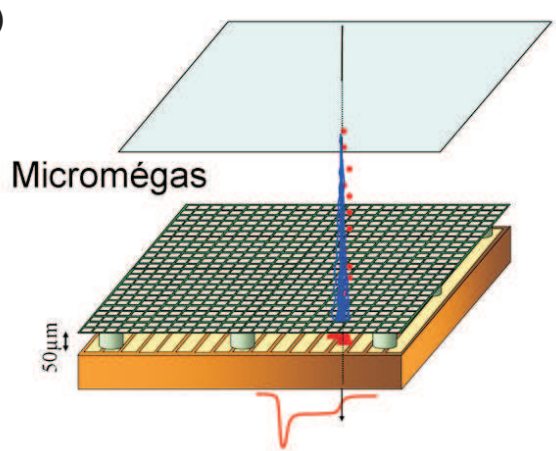

b)

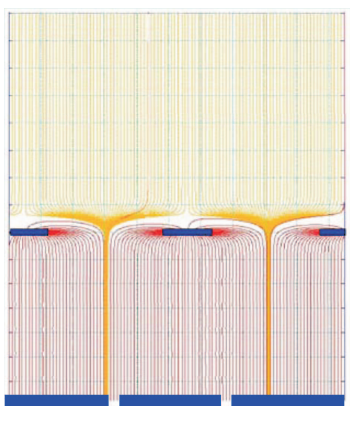

c)

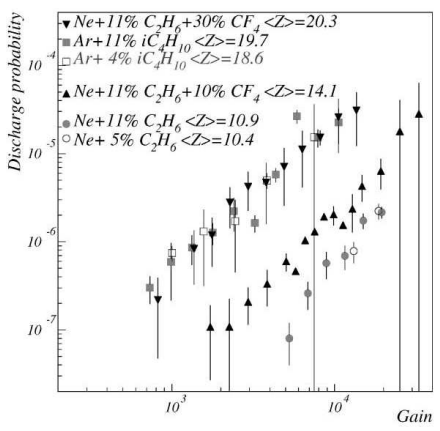

Figure 2: Micromegas: a) Schematic drawing of a Micromegas detector (from http://www.linearcollider.org), b) electric field configuration of the Micromegas detector, c) discharge probability in high-rate hadronic beams.

produced in the gas amplifications could be significantly faster neutralized and a higher particle flux could be detected (see figure 1b). Unfortunately, the thin strips proved to be very sensitive to destructive discharges. Especially those induced by high charge densities, i. e. from highly ionizing particles, could remove a part of the strip from the substrate resulting in dead areas or, even worse, producing a local shorts between anode and cathode (see figure 1c). Besides, many experiments showed that MSGCs were prone to aging. A thin insulating polymer film was found to build up with charge accumulation. This film modifies the electric field above the anode strip resulting in lower gas gains.

To remedy these unwanted side effects of miniaturization, a number of different electrode geometries were suggested and tested: Microgap Chambers[8], Microdot Chambers[9], and MicroPics[10] are some examples. However, the two most commonly used devices, Micromegas[11] and Gas Electron Multipliers[12], have proven to run reliably even in high-rate hadronic beams (e. g. at the COMPASS experiment [13]).

\section{Micromegas}

The gas amplification in Micromegas detectors is based on the principle of parallel plates: A thin mesh is stretched at a small distance (typically 50-100 $\mu \mathrm{m}$ ) above the readout anode (see figure 2a). If a high electric field is applied across this gap, and a low electric field is applied to the drift region, electrons will drift towards the mesh, are then focused into the mesh holes and are multiplied in the high field region (see figure 2b). Due to the parallel field configuration and the saturation of the Townsend coefficient at high electric fields, the Micromegas have demonstrated excellent gain stability and high rate capability. During data taking at the CAST-experiment it was shown that the energy resolution is about $16 \%$ (FWHM at $5.9 \mathrm{keV}$ ) and the stability is also very good (see reference [14]). For example the gas gain showed over a period of several months variations of only $10 \%$ due to changes in environmental conditions, such as atmospheric pressure or temperature. These effects could be calibrated with radioactive sources.

Since the thin gap of the Micromegas allows only very little diffusion, the spatial extension of the signal is very small. In many applications this turned out to be a limiting factor of the 

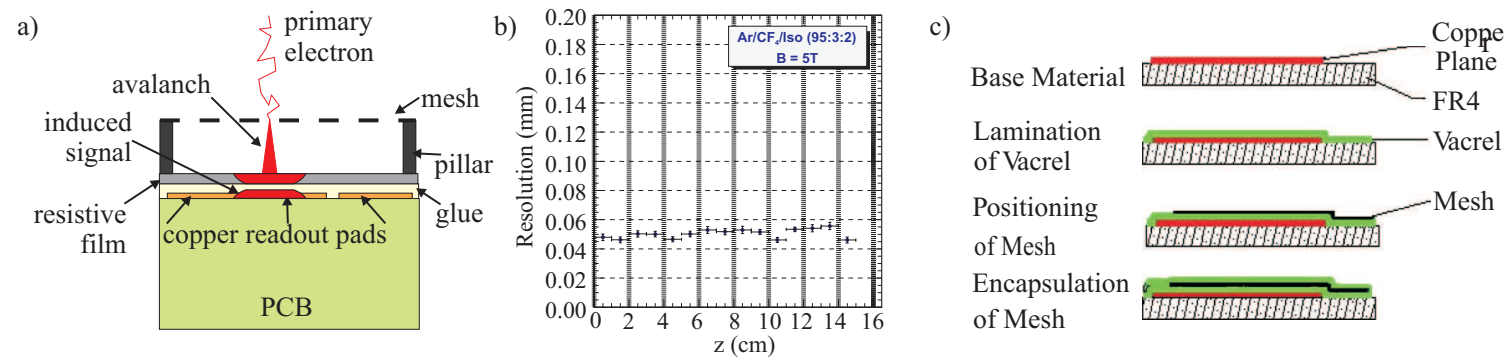

Figure 3: Micromegas: a) schematic drawing of a Micromegas and resistive foil on readout electrodes, b) spatial resolution of the Micromegas with resistive foils, c) bulk-Micromegas [17]).

spatial resolution, if the readout pad size can not be reduced sufficiently due to electronic package densities and readout speed considerations. Applying a resistive foil on top of the readout electrode can improve the spatial resolution. As shown in figure 3a the small electron signal is spread over a larger area, so that several pads can pick up the signal and a center of gravity algorithm can determine a precise location of the primary charge (see figure $3 b$ ). This method is described in detail in reference [16].

Initially some applications of Micromegas suggested that highly ionizing particles or very high rates of minimal ionizing particles are likely to trigger discharges from the mesh to the readout electrodes. In contrast to the MSGCs these discharges are not destructive to the detector, but introduce a significant dead time and pose a risk to the readout electronics. This has been studied in detail and the discharge probability could be significantly reduced by optimizing the production process (see figure 2c). For example the roughness of surfaces such as the readout electrode were identified as a main source of discharges. Covering the anode with the aforementioned resistive foil removes any roughness and therefore also increases the stability.

To allow the simple production of large areas a new manufacturing technique was pioneered (see reference [17]). The new detector type goes under the name of bulk-Micromegas and the production flow is shown in figure 3c. The readout area, usually a printed circuit board (PCB), is covered with a photoresistive film (e. g. Vacrel) of the same thickness as the gap. Then a commercially available woven wire mesh is placed atop and encapsulated with a second layer of photoresist. By a photolithographic method the photoresistive material is etched to produce the pillars supporting the mesh. Due to their simplicity and good features, bulk-Micromegas are already used in a number of applications. For example the near detector of the T2K experiment uses a Time Projection Chamber (TPC) with bulk-Micromegas readout.

\section{Gas Electron Multipliers}

The Gas Electron Multipliers (GEMs) are made of a $50 \mu$ m-thick kapton foil covered on both sides with a thin copper layer. A hexagonal pattern of double-conically shaped holes with a diameter of $60-70 \mu \mathrm{m}$ is etched at a pitch of $140 \mu \mathrm{m}$ into this sandwich structure. If a voltage difference of typically $300-400 \mathrm{~V}$ is applied to both electrodes, a strong electric dipole field is generated inside the holes. Electrons in the drift volume are guided into the holes, where they are multiplied, and a large fraction of those are released into the volume below the GEM. Due to the decoupling of gas 

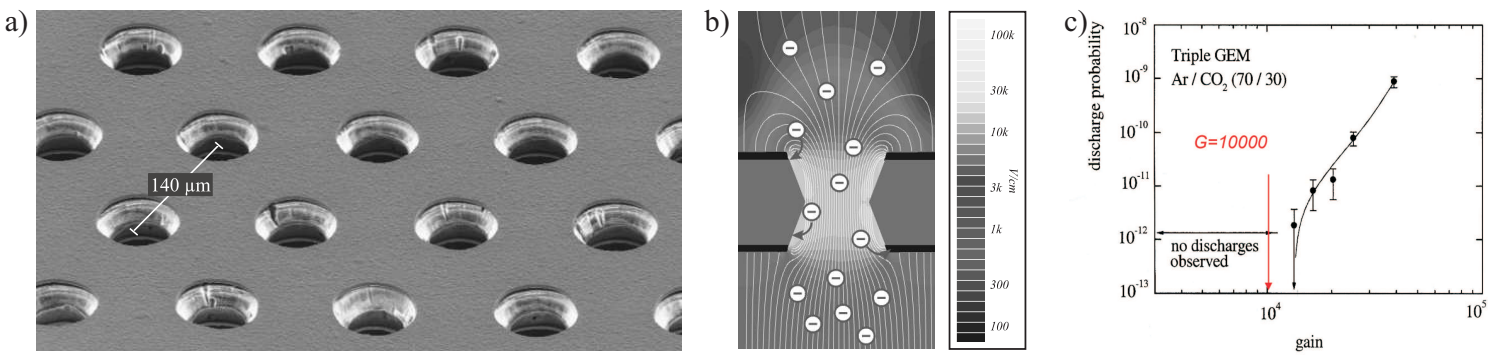

Figure 4: Gas Electron Multiplier: a) SEM picture of the device, b) schematic drawing of electric field lines in GEM-holes, c) discharge probability in high rate hadronic beams.

amplification and readout structure, the GEM is more flexible than most MPGDs and in particular a multi-stage gas amplification is possible by stacking several GEMs on top of each other. In this way lower voltage differences can be applied to the individual GEMs. Furthermore, the diffusion between the GEMs spreads the charge over several holes, thus lowering the charge density in the multiplication region. Therefore, the discharge probability is considerably below the one of other MPGDs.[19]

Despite these excellent results an intrinsically spark protected device called RETGEM has been designed. For this the copper was replaced by a resistive paste, that was cured before the holes were added. Due to the high resistivity (in the order of $1 \mathrm{M} \Omega / \mathrm{cm}$ ), the flow of electrons is hindered, and thus the development of a spark is inhibited from the beginning.[20]

Covering large areas with GEM foils is studied in the context of upgrades of large high energy physics experiments. The procurement of industrially produced GEMs is an important ingredient of this. Therefore, GEMs etched by a company called Tech-Etch have been compared with the one produced at CERN, and both GEM types have shown comparable performance.[21]

In the following three important applications shall be discussed with one example each:

\subsection{Thin Tracking Detectors}

MPGD with a thin drift volume of only a few millimeter have been used in many experiments. In the following an R\& D project for a tracker upgrade of the aforementioned COMPASS experiment shall be presented [22]:

The original GEM-tracker spares the central part of the detector, where the unscattered high rate beam of the SPS passes through. To increase the acceptance of the detector, R\& D-detectors were built to test their behavior at particle rate up to $5 \cdot 10^{7}$ particles $/ \mathrm{s}$. 1000 square pads of $1 \mathrm{~mm}^{2}$ are used to readout the central part of the detector. This areas is surrounded by 1000 strips covering a total area of $10 \times 10 \mathrm{~cm}^{2}$. The remaining layout of the detectors follows closely the layout of the COMPASS GEM detectors already in use. With this setup a test beam at the CERN SPS was conducted comparing the performance of the detector at low particle rates $\left(1.2 \cdot 10^{4}\right.$ particles $\left./ \mathrm{mm}^{2} / \mathrm{s}\right)$ and at high particle rates $\left(1.2 \cdot 10^{5}\right.$ particles $\left./ \mathrm{mm}^{2} / \mathrm{s}\right)$ : The efficiency dropped from $98.5 \%$ to $96 \%$, while the spatial resolution and time resolution degraded from $90 \mu \mathrm{m}$ to $135 \mu \mathrm{m}$ and from $6.3 \mathrm{~ns}$ to $7.3 \mathrm{~ns}$ respectively. These results fulfill the requirements and the upgrade will therefore be carried out. 
a)

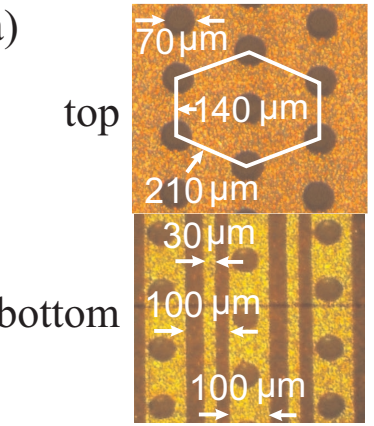

b)

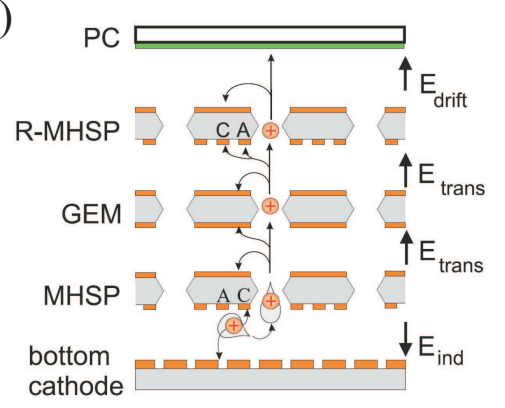

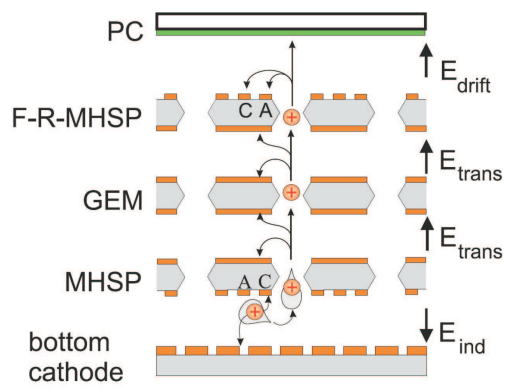

Figure 5: Micro-Hole \& Stip plates: a) photograph of both electrodes, b) schematic drawing of detector setup with GEMs, MHSP, and R-MHSP, c) schematic drawing of detector setup with GEMs, MHSP, and the flipped R-MHSP F-R-MHSP.[24]

\subsection{Photon Detection}

GEM detectors are well suited for photon detection, if the cathode is covered with a photosensitive layer: Electrons knocked out of the photocathode by photons will then drift towards the GEM-stack and will be amplified. It was demonstrated that the electron collection and amplification is performed with close to $100 \%$ efficiency. It is, however, crucial to suppress the ions generated during the gas amplification, as far as possible, since ions released into the drift volume can hit the sensitive photocathode with sufficient energy to liberate extra electrons (secondary electron emission), thus setting of a constant chain of signals.

It was demonstrated, that by changing the field configuration of a GEM, the ion backflow (IBF) could be lowered. Figure 5a shows a Micro-Hole \& Strip Plate (MHSP), which introduces on one side of the GEM an additional cathode strip. This strip can be placed at a higher voltage than the surrounding area, representing an additional gas amplification stage similar to the MSGCs. In this setup the gas gain in the GEM holes can be reduced, thus lowering the ion production. On the other hand, if used as the topmost gas amplification stage, the strips can be placed at a lower voltage than the surrounding area, attracting the ions produced in the lower gas amplification stages (see figure 5). This setup is called Reverse-MHSP (R-MHSP) and an IBF as low as $4 \cdot 10^{-4}$ was reported.[23] Flipping the topmost R-MSHP, the IBF could be reduced to $1.5 \cdot 10^{-4}$ at a gas amplification of $10^{4} \cdot[24]$

\subsection{Large Volume Tracking Devices: Time Projection Chamber}

The combination of a Time Projection Chamber with MPGDs is very attractive, since the new readout overcomes several of the shortcomings conventional TPC with MWPC readout revealed. For one thing, the intrinsic ion backflow suppression of the MPGD is very attractive since it allows the reduction of the ions escaping into the drift volume to a minimum. What is more, some effects limiting the spatial resolution in MWPC devices do not exist anymore. Here the $E \times B$-effect at the vicinity of the wires, the wide induction signals on the pads, as well as long signal of the ion tail should be mentioned.

Therefore, a number of smaller and larger experiments are studying the use of MPGD based TPCs, e. g. currently two of the three detector concepts for the International Linear Collider are 
a)

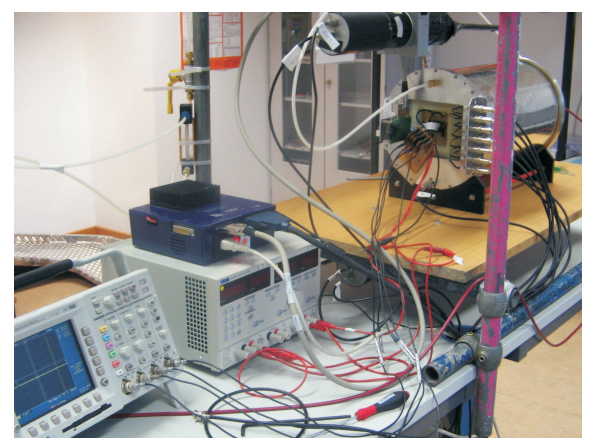

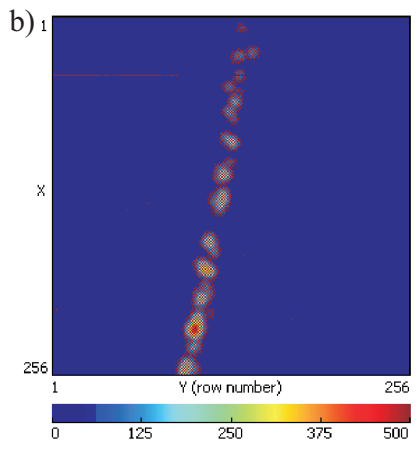

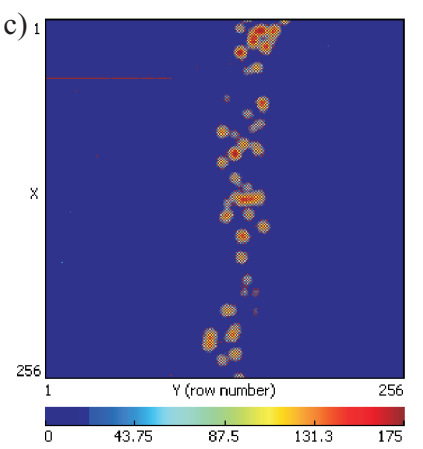

Figure 6: Prototype detector of a TPC with triple GEM and Pixel-ASIC readout: a) photograph of a cosmic ray test setup, b) signal of track passing through the detector close to the readout, c) close to the cathode.

studying such a device as central tracker and a large number of studies have been performed.

\section{Readout with highly pixelized ASICs}

Many experiments have shown that the spatial resolution of MPGDs is limited by the pad size. In particular the angular pad effects limits the spatial resolution of tracks passing at an inclination with respect to the long axis of a rectangular pad.[25]

To decrease the pad size as much as possible, but to keep up with the increasing amount of electronic channels at the same time, the use of ASIC chips has been tested. Especially the Timepix chip [26], a derivate of the Medipix2, has been chosen as a good candidate. The chip has originally been developed to be operated with a solid state sensor, each pixel being bump-bonded to the sensor. For gaseous detectors the bare ASIC is placed directly below the MPGD stage. Its bump bonding pads are used to collect the electron signal of the Micromegas or lowest GEM.

\subsection{Timepix readout of GEM Signals}

At Bonn a prototype detector of a TPC with triple GEM and Timepix readout was setup and tested with cosmic rays (see figure 6a) and in a $0.5 \mathrm{GeV}$ electron beam of the ELSA accelerator. Figure 6 shows signals originating from tracks passing close to the anode (b) and close to the cathode (c). The charge depositions seen in both events correspond to the amplified signal of single or multi-electron clusters. The transverse spatial resolution was determined in dependence on the drift distance as shown in figure 7a. For single electrons, a square root rise $\left(D_{t} \sqrt{z}\right)$ is expected due to diffusion. Threshold effects and other limitations (see below) introduce a constant offset $\sigma_{0}$. Finally, the declustering of multi-electron clusters due to diffusion could be observed, resulting in a net number of electrons per charge deposition parameterized by $n_{e l e}=1+a e^{b z}$. Therefore, a fit function

$$
\sigma=\sqrt{\sigma_{0}^{2}+\frac{D_{t}^{2}}{n_{\text {ele }}} z}
$$

was used to describe the data with good agreement. During the test beam the transverse spatial resolution was studied in dependence on the track inclination in the pad plane. Figure 7 shows 
a)

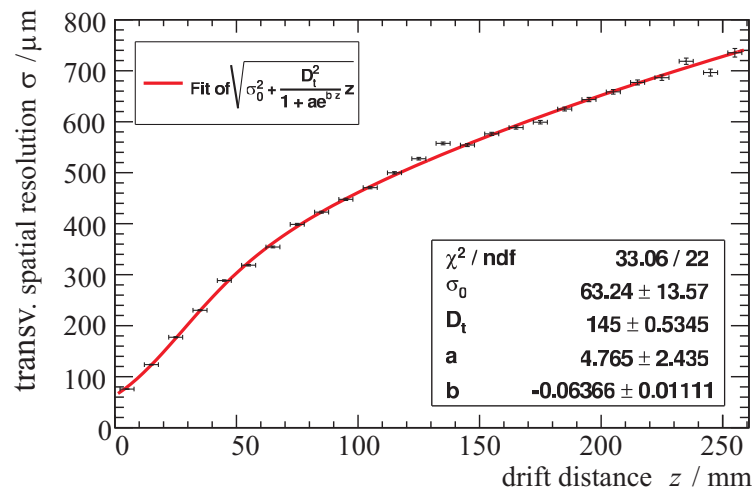

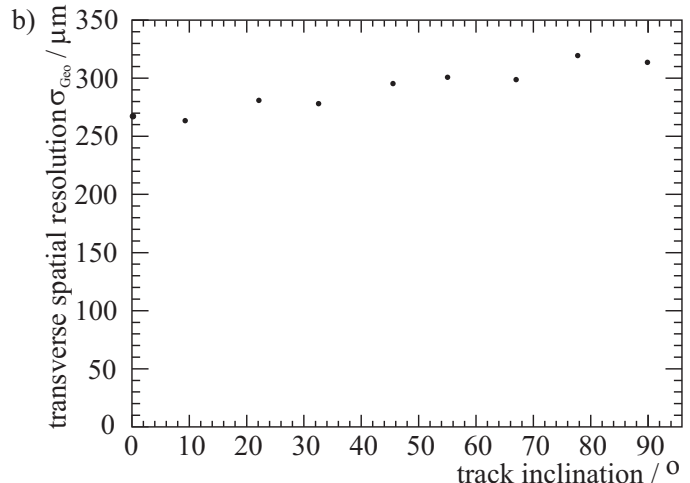

Figure 7: TPC with triple GEM and Pixel-ASIC readout. Transverse spatial resolution in dependence on: a) drift distance, b) track inclination in the readout plane.[27]

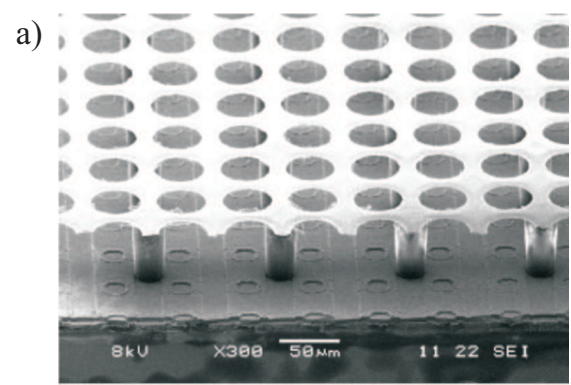

b)

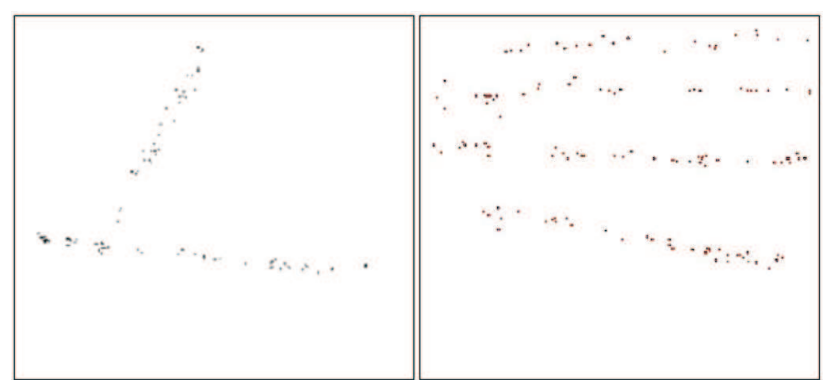

Figure 8: InGrid-detector: a) SEM picture of detector, b) and c) 2 dimensional pictures of tracks of highenergy ionizing particles crossing the detector.[29]

quasi no dependence on the track inclination. The small systematic increase towards larger angles is mostly due to a shift in electron energy caused by experimental setup.

It was also demonstrated that in this setup the component limiting the transverse spatial resolution was the hole pattern of the GEMs: Since the electrons were forced into the hexagonal hole pattern with a pitch of $140 \mu \mathrm{m}$, this spatial localization is a significant contribution to the constant offset $\sigma_{0}$.

\subsection{InGrid}

To match the pitch of the gas amplification stage with the one of the readout chip a Micromegas was adopted as gas amplification stage. This combination of Micromegas and ASIC is called InGrid. The mesh is built in a post-processing step similar to the production of bulk-Micromegas and consists of $50 \mu \mathrm{m}$ high SU8-pillars and a $0.8 \mu \mathrm{m}$ thick aluminum plane with holes that are aligned with the pixels of the Timepix chip (see figure 8 and references [28] and [29]).

For Micromegas the gas amplification avalanche of a single primary electron is collected by a single pixel, whereas it is spread over several tens of pixels in the case of a triple GEM amplification stage. The smaller signal spread results in larger signals per pixel and thus, smaller gas gains are necessary. On the other hand, the spatial resolution of single electrons is given by pixel size $/ \sqrt{12}$, since no center of gravity or similar algorithm is possible. Another drawback is that each pixel of the Timepix chip can record either the time of arrival or the integrated charge. Thus, only one of the 
two information can be obtained with InGrid detectors for now. To remedy this effect, a successor chip will be designed in the near future and is planned to be available in 2010 .

Similar to the standard Micromegas, initial test showed that InGrids are vulnerable to discharges triggered by high charge densities. To prevent the large charge depositions from destroying the ASIC and to reduce the number of discharges, a thin layer of amorphous $\mathrm{Si}$ was placed on top of the readout chip before building the mesh. This high resistive material $\left(10^{11} \Omega / \mathrm{cm}\right)$ helps to quench discharges by a local reduction of the electric field due to the build up of a surface charge.[30] Preliminary tests with slow $\alpha$-particles show excellent chip protection.

One application of the InGrid-technology is the GOSSIP-detector. Here a very thin gas gap of 2-3 mm only is placed atop the InGrid. In this configuration minimal ionizing particles traversing the detector perpendicular to the ASIC have a high probability to create 2-3 primary clusters, which can be amplified and detected by the InGrid detector. This setup could be used as low mass vertex detector in high energy physics experiments.

\section{Summary}

The R\& D on Micropattern Gas Detectors is a very active field. Many new ideas have been put forward and tested. Especially the first results from the readout with highly pixelized ASIC chips look very promising.

The formation of a new R\& D collaboration at CERN (RD51) opens the opportunity for good communication of different groups and gives better accessibility to much needed but rare infrastructure such as test beam, high magnetic fields and irradiation facilities.

\section{References}

[1] E. Rutherford, H. Geiger, Proc. Royal Soc. A81 (1908), 141.

[2] H. Geiger, W. Müller, Phys. Zeits. 29 (1928) 839.

[3] G. Charpak, Proc. Int. Symp. Nuclear Electronics (Versailles 10-13 Sept 1968).

[4] A. Oed, Position sensitive detector with microstrip anode for electron multiplication with gases, Nucl. Instrum. Meth. A 263 (1988) 351.

[5] P. Colas et al., The readout of a GEM- or micromegas-equipped TPC by means of the Medipix2 CMOS sensor as direct anode, Nucl. Instrum. Meth. A 535 (2004) 506.

[6] R. Bellazini et al., Reading a GEM with a VLSI pixel ASIC used as a direct charge collecting anode, Nucl. Instrum. Meth. A 535 (2004) 477.

[7] F. Sauli, A. Sharma, Micropattern Gaseous Detectors, Annu. Rev. Nucl. Part. Sci. 1999. 49:341-88.

[8] F. Angelini, et al., The micro-gap chamber, Nucl. Instrum. Meth. A 335 (1993) 69.

[9] S. F. Biagi, T. J. Jones, The microdot gas avalanche chamber: an investigation of new geometries, Nucl. Instrum. Meth. A 361 (1995) 72.

[10] A. Ochi et al., A new design of the gaseous imaging detector: Micro Pixel Chamber, Nucl. Instr. and Meth. A 471 (2001), 264.

[11] I. Giomataris, et al., MICROMEGAS: a high-granularity position-sensitive gaseous detector for high particle-flux environments, Nucl. Instrum. Meth. A 376 (1996) 29. 
[12] F. Sauli, GEM: A new concept for gas amplification in gas detectors, Nucl. Instrum. Meth. A 386 (1997) 531.

[13] The COMPASS Collaboration, COMPASS: A proposal for a COmmon Muon and Proton Apparatus for Structure and Spectroscopy, CERN/SPSLC 96-14, SPSC/P 297 (1996).

[14] P. Abbon et al., The Micromegas detector of the CAST experiment, New J. Phys. 9170.

[15] D. Thers et al., Micromegas as a large microstrip detector for the COMPASS experiment, Nucl. Instr. and Meth. A 469 (2001) 133.

[16] M. Dixit et al., Micromegas TPC studies at high magnetic fields using the charge dispersion signal, Nucl. Instr. and Meth. A 581 (2007) 254.

[17] I. Giomataris et al., Micromegas in a bulk, Nucl. Instr. and Meth. A 560 (2006) 405.

[18] P. Baron et al. Large bulk-MicroMegas detectors for TPC applications in HEP, 2007 IEEE Nuclear Science Symposium Conference Record MP4-3.

[19] S. Bachmann et al., Performance of GEM detectors in high intensity particle beams, Nucl. Instr. and Meth. A 470 (2001) 548.

[20] G. Agócs et al., Developments and the preliminary tests of resistive GEMs manufactured by a screen printing technology, JINST 3 (2008) P02012.

[21] F. Simon et al. Development of Tracking Detectors With Industrially Produced GEM Foils, IEEE Trans. Nucl. Sci. 54, Issue 6, (2007) 2646.

[22] B. Ketzer et al., A Triple-GEM Detector with Pixel Readout for High-Rate Beam Tracking in COMPASS, 2007 IEEE Nuclear Science Symposium Conference Record N12-5.

[23] A.V. Lyashenko et al., Advances in ion back-flow reduction in cascaded gaseous electron multipliers incorporating R-MHSP elements, JINST 1 (2006) P10004.

[24] A.V. Lyashenko et al., Further progress in ion back-flow reduction with patterned gaseous hole multipliers, JINST 2 (2007) P08004.

[25] J. Kaminski et al., Study of Various Anode Pad Readout Geometries in a GEM-TPC, IEEE Trans. Nucl. Sci. 53 (2006) 2936.

[26] X. Llopart, et al. Timepix, a 65k programmable pixel readout for arrival time, energy and/or photon counting measurements, Nucl. Instr. and Meth. A 581 (2007) 485.

[27] J. Kaminski et al., Time Projection Chamber with Triple GEM and Pixel Readout, 2008 IEEE Nuclear Science Symposium Conference Record N43-7.

[28] M. Chefdeville et al., An electron-multiplying 'Micromegas' grid made in silicon wafer post-processing technology, Nucl. Instrum. Meth. A 556, (2006) 490.

[29] V. M. Blanco Carballo et al., A Radiation Imaging Detector Made by Postprocessing a Standard CMOS Chip, IEEE Electron Device Letters 29, NO. 6, (2008) 585.

[30] H. van der Graaf, GridPix: An integrated readout system for gaseous detectors with a pixel chip as anode, Nucl. Instrum. Meth. A 580, (2007) 1023. 